\title{
Hacking History: Redressing Gender Inequities on Wikipedia Through an Editathon
}

\author{
Dr Nina Hood ${ }^{1}$ and Prof Allison Littlejohn² \\ 1 University of Auckland, 2 Open University, UK \\ *corresponding author
}

\begin{abstract}
Editathons are a relatively new type of learning event, which enable participants to create or edit Wikipedia content on a particular topic. This paper explores the experiences of nine participants of an editathon at the University of Edinburgh on the topic of the Edinburgh Seven, who were the first women to attend medical school in 19th century United Kingdom. This study draws on the critical approach to learning technology to position and explore an editathon as a learning opportunity to increase participants' critical awareness of how the Internet, open resources, and Wikipedia are shaping how we engage with information and construct knowledge. Within this, there is a particular focus on recognising persisting gender inequities and biases online. The qualitative interviews captured rich narrative learning stories, which traced the journey participants took during the editathon. Participants transformed from being online information consumers to active contributors (editors), prompting new critical understandings and an evolving sense of agency. The participants' learning was focused in three primary areas: (1) a rewriting of history that redresses gender inequities and the championing of the female voice on Wikipedia (both as editors and subject matter); (2) the role of Wikipedia in shaping society's access to and engagement with information, particularly information on traditionally marginalised subjects, and the interplay of the individual and the collective in developing and owning that knowledge; and (3) the positioning of traditional media in the digital age.
\end{abstract}

Keywords: editathon, critical approach, gender, Wikipedia, learning 


\section{Background}

Editathons are a relatively new type of learning event, which enable participants to create or edit Wikipedia content on a particular topic. Events enable budding editors to learn together at a scheduled time, often in a designated physical location. They introduce participants to the Wikipedia community, supporting the development of new skills and knowledge, and often include basic editing training. Frequently, editathons have a secondary purpose of addressing biases within Wikipedia by raising awareness of the gender, cultural, and geographic disparities that affect both the content and the editing community on Wikipedia (Collier \& Bear, 2012; Hargittai \& Shaw, 2015). As such, editathons support new forms of knowledge construction, which allow opportunities for the democratisation of knowledge (Knorr-Centina, 2007, 2008) and trigger new roles and accountabilities around how knowledge is created.

This paper focuses on an editathon that took place in 2015 at the University of Edinburgh on the topic of the Edinburgh Seven, the name given to the first group of women to studied medicine at the University. The primary purposes of the event were to develop among participants an understanding of the community norms and rules governing Wikipedia, and to build their technical know-how and confidence to edit Wikipedia entries. This study was designed to explore the editathon as an informal, professional learning event. Eraut (2000) positions informal learning as learning that is not planned around or structured by a bounded course, imposed learning outcomes, or formal assessment, but instead driven by the motivations and agency of individual learners who navigate their own learning journey. Participation in the editathon was voluntary, with individuals determining the nature and level of their engagement throughout event. There were no expectations around how much or even if they would edit or contribute new content to Wikipedia.

This study was initially conceived as a project to explore the employment of social network analysis [SNA] to trace the contributions of individual participants during and after the editathon event. Qualitative interviews with nine of the 47 participants were undertaken to explore in more detail the editathon as a learning event. They focused particularly on how participants self-organised to facilitate open information exchange and how participants accumulated knowledge during the event. The interviews provided insight into the learning, which moved beyond the scope of the original project. Embedded within the narratives of the nine interviewees was an evolving understanding of the ways in which the Internet and digital media shape the information with which they engage and how they interpret and utilise this information to construct particular historical narratives. The participants also discussed an emerging sense of agency as they not only recognised prevailing norms of online representation and behaviour, but also actively addressed and redressed these.

The recognition of these themes within the participants' narratives prompted a critical re-reading of the editathon. This re-reading explored how the topic of the editathon combined with participants' transitioned from consumers of information to knowledge producers provoked new insight into their contexts of operation - historical, institutional, professional, and personal. It explores the potential of an informal learning event to provoke new understandings and the adoption of new roles by participants, and to raise awareness of how the non-neutral construction of knowledge and artefacts on the Internet permeates our understandings and constructs particular realities, of which, too often, we are not actively cognisant. 


\section{Literature Review}

\section{Wikipedia}

Wikis, such as Wikipedia, are edited by a number of individuals who keep track of the changes and adaptations that are being made. Their ongoing development and growth, therefore, is reliant on the co-construction of content by a community of editors who collectively take ownership for contributing and updating information.

These new social digital tools have transformed information production and distribution by requiring people to take on new roles and responsibilities, raising questions around how information is generated and produced (Fenwick, Nerland, \& Jensen, 2012; Knorr-Cetina, 2007). Ebersbach and Glaser (2004) argue that wikis are predicated on decentralised, egalitarian structures that offer individuals the flexibility and opportunity to engage with, and contribute to, the wikis in their own personal way. While offering potentially new opportunities for information construction, and theoretically opening up information creation and dissemination to a much broader population than previously has been possible using traditional media, research suggests that most user-generated content on the Internet conforms to pre-existing economic, social, and political models (Manovich, 2009). The reported systematic and structural biases exist in spite of Wikipedia's espoused neutral point of view policy, which states "All encyclopaedic content on Wikipedia must be written from a neutral point of view [NPOV], which means representing fairly, proportionately, and as far as possible, without editorial bias, all of the significant views that have been published by reliable sources on a topic" (Wikipedia, 2016).

\section{Women and Wikipedia}

Gender biases are apparent in both the presentation and production of content on Wikipedia, as well as in the gender distribution of contributors to Wikipedia. Research suggests that only 8 to $18 \%$ of editors on Wikipedia are female (Antin, Yee, Cheshire, \& Nov, 2011; Cohen, 2011; Collier \& Bear, 2012; Glott, Ghosh, \& Schmidt, 2010; Hill \& Shaw, 2013; Lam et al., 2011; Wikimedia Foundation, 2011). The gender divide exists not only in terms of absolute numbers but also in the treatment of women editors. Lam et al. (2011) found that women are more reverted than men (their contributions are discarded), while Collier and Bear (2012) report that women's lower levels of contribution result from aggressive behaviour towards them.

The (re)presentation of women on Wikipedia also differs substantially from that of men. Biographies of women are less well-developed and male editors are less likely to edit women's biographies (Reagle \& Rhue, 2011). Studies also have found that the use of language varies between biographies of men and women (Graells-Garrido, Lalmas, \& Menczer, 2015; Wagner, Graells-Garrido, Garcia, \& Menczer, 2016). Wagner et al. (2016) determined that articles on women were more likely to include gendered words like "women," "female," and "lady" compared with articles about men that rarely use gendered words such as "man," "male," or "gentleman." Graells-Garrido, Lalmas, and Menczer (2015) similarly found that women were more likely to be associated with gendered words, and in particular, entries on women were strongly associated with "her husband" and "first woman." Amanda Filipacchi, in a widely cited 2013 opinion piece in The New York Times, reported the editorial decision of women being removed from the American Novelists category and moved to a subcategory for American Women Novelists. 
This positioning and treatment of women (both as subjects and editors/contributors) on Wikipedia mirrors trends identified in other online environments. For example, gender inequity is present in the most popular political blogs (Harp \& Tremayne, 2006) and sexism and misogyny continues to prevail in mainstream and social media. While some commentators have suggested that one might expect greater gender equity online because of its "openness" and the apparent ease of entry, as Couldry (2012) reminds us “"we perform identity and develop public or quasi-public profiles within the constraints of platforms ... as a result, we risk a deep penetration by market logics into the very lineaments of self-reflection and self-expression" (p. 57). Dominant discourses and the continued marginalization of traditionally excluded voices and histories prevails in the online environment. However, Shaw (2014) suggests that at their most powerful digital tools allow groups to produce new forms of knowledge and posit counter-discourses.

\section{Context and Methods}

\section{Theoretical Framework}

This study draws on the critical approach to learning technology to position and explore an editathon as a learning opportunity to increase participants' critical awareness of how the Internet, open resources, and Wikipedia are shaping how we engage with information and construct knowledge. The critical approach emphasises the positioning of learning and technology within its broader organisational, political, economic, and social contexts in order to explore how it can foster, support, and counteract issues of empowerment, equality, and social justice (Bakardijieva \& Smith, 2001; Gunter, 2009; Selwyn, 2008, 2010). Bakardijieva and Smith (2001) suggest the potential for individuals to develop new agency when engaging with the Internet, and the ability to contribute actively to, and to generate new interpretations of, technology in order to promote democratic, feminist, or revisionist history aims. Oliver (2011) builds on these ideas positioning the critical approach as facilitating a movement beyond the immediate context of learning gains or patterns of interaction, to question the broader positioning and role of technology and how it is shaping both individual lived experiences and system-wide expectations, patterns of behaviour, and modes of thinking.

The adoption of the critical approach allows this study to move beyond its initial scope of exploring the socially collaborative nature of learning in an editathon and the tracking of the learning process through the network social ties. The critical approach enables an exploration of participants' adoption of new roles and new agency and how these are positioned within their wider experiences of the Internet, learning, and Wikipedia. That is, an examination of participants' experiences of an editathon through the lens of what Selwyn (2010) terms the social milieu of technology use.

Re-analysing participants' accounts utilising a critical framework enables an exploration of how an editathon can build new capacity in participants as they transition from consumers and users of online material to producers of that material. This active awakening of new understandings and repositioning of the individual connects with Mellucci's (1996) notion of cognitive liberation. That is, through engaging in particular activities - both as an individual and as part of a wider group - an individual gains awareness of a broader movement (the marginalisation of women online) and reframes themselves - their beliefs and activity - in relation to this new understanding, and as such 
joins a collective movement to redress inequity online. This opens possibilities for a cyberfeminist reading of participants' learning journeys, as they recognise how the Internet and their actions as knowledge producers provide avenues to liberate (or oppress) women (Shaw, 2014).

\section{Context}

This study is situated within an editathon event on the theme of the Edinburgh Seven, the first women to study medicine at the University of Edinburgh. The event took place at the University of Edinburgh and was led by the University's Information Services in conjunction with the School of Literature, Languages and Cultures, the Moray House School of Education, EDINA, and the National Library of Scotland. The editathon was open to everyone, but particularly targeted students, university staff and faculty, and members of the public who had an interest in developing their knowledge of Wikipedia.

The editathon was held over four afternoons in a large, interactive learning classroom. Participants determined how much of the event they attended as well as their level of engagement. A total of 47 participants engaged in online editing. During the event participants had access to library archivists and media specialists, academic colleagues and Wikimedia experts, including a Wikimedian trainer in residence, who provided training on how to edit Wikipedia and participate in an open knowledge community. Many of these people functioned in the dual role of expert teacher and fellow editathon participant. Participants also were able to access a range of artefacts including archived materials such as newspaper reports and photographs, books, and online sources.

\section{Methods}

Following the editathon event, nine participants were invited to partake in an interview to discuss their experiences of the event. The participants were purposively selected using the quantitative data that traced the online edits of the 47 active editathon participants. This analysis exposed the wiki pages that each of the participants had made edits to, enabling insight into both the activity level of each participant (Littlejohn \& Hood, 2018). Two participants were central in the online network, two had been active in terms of minor changes, two had been active on wiki pages not covered by others, one had not made any edits, and two were co-ordinators of the event.

The one-hour interviews were conducted via Skype using a semi-structured instrument. During the interview participants were asked to comment on their experience of the editathon, including what and how they had learned during the event, their engagement with other participants, and their behaviour and activity since the editathon. Participants were also shown the network analysis diagrams and discussed their node and level of interactivity. The interviews were audio-recorded and transcribed verbatim.

The original data analysis process was focused on constructing an individual learning profile for each of the interviewees, which enabled comparisons to be drawn both between the SNA and interview data, and between the knowledge construction behaviours of the nine participants (Littlejohn \& Hood, 2018). However, during the first two-coding round, which developed the content and first thematic codes (Miles \& Huberman, 1994), the emergence of content and themes that lay outside the original scope of the study emerged from the data. Embedded within the participants' narrative accounts was a developing understanding of the ability of an editathon to prompt changes to the ways that participants conceptualised and engaged with Wikipedia and the Internet more generally, and the development of new agency among participants for the role they could play in repurposing Wikipedia, 
rewriting history, and reclaiming traditional media in the digital age. These observations of the data prompted a new analysis process, which laid a critical lens over the data. A third round of data analysis, separate from the original analysis process, was conducted. Four new thematic codes were developed: 1. the rewriting of women in history, 2. the role of Wikipedia in shaping access to and presentation of information, 3. the role of traditional media in a digital world, and 4. and the developing sense of agency and ownership among participants for the topic and constructing historical accounts.

\section{Findings and Discussion}

The findings presented below explore how participants created new meanings, adopted new roles, and developed new social relations through their participation in the editathon and as they developed into their new roles as editors. It considers how adopting the role of editor and the new understandings and shift in perspective this brought, contributed to new interpretations and evolving engagement with the Internet. The interviewees' accounts of their participation in the editathon are discussed here in relation to three themes: 1. rewriting history and the development of the female voice on Wikipedia, 2. the role of Wikipedia in shaping society's access to and engagement with information and the interplay of the individual and the collective in developing and owning that knowledge; and 3. the positioning of traditional media in the digital age.

\section{Becoming Editors and Rewriting History}

As the participants grew into their new roles as Wikipedia editors they came to realise the responsibility they had to represent history and to shape how others engage with historical information. The participants described an evolving realisation that: (a) previously the historical actions of the Edinburgh Seven women either were not available or (largely) had been interpreted and represented in a biased way; (b) they held responsibility for disseminating their interpretation of what the women had achieved; and (c) they struggled with how to represent the women in the social media space.

For a majority of interviewees, the primary motivation for their participation in the editathon was to develop practical, technical knowledge about how to contribute to and edit Wikipedia entries. Eight out of the 10 participants interviewed knew little about the topic of the editathon before the event. While the subject did not drive initial engagement for many interviewees, it emerged as a recurring theme running through the narratives of their learning journeys as the participants became aware of their new roles as the writers and recorders of the history of the Edinburgh Seven.

The Edinburgh Seven became the specific example through which participants could develop their understanding of the place and (re)presentation of women in history, and the role the Internet plays in perpetuating male-dominated historical narratives. This intersection between the specific topic of the editathon and the construction of a more macro-level understanding of the continuation of the traditional male-dominated discourse on the Internet, together with the behaviour that can accompany this dominance, permeated participants' narratives. Emma reflected that the "behaviour of people in protecting Wikipedia, maybe over zealously protecting it, or being sexist about it, which was interesting in relation to the topic itself," while Louise commented on lack of existing online material or information on the women of the Edinburgh Seven. 
Through actively creating content for Wikipedia, participants developed new understandings of how the presentation of media on the Internet shapes the meanings and interpretations consumers of information construct. Nick discussed the (negative) discourses that can be attached to female figures:

The person I was writing a page on it was much easier to find information about the fact that she'd accused some people of witchcraft as a child than it was about her history as an innovator and technologist in the thread industry and where you saw one you didn't usually see the other one connected, but it does seem to be the same person.

The editathon not only prompted new understandings of how particular stories and messages become associated with female historical figures, but also the power of different digital objects to promote and perpetuate particular historical narratives. The merging of text and image on the Internet plays an important role in shaping understandings of events and people. Justine described her growing appreciation of how images inform historical accounts:

She does look like a battle axe in the picture and it is the picture that you often use, but then I had this book and there was a very nice picture of her and I was thinking why is this other picture always used and why shouldn't it be this one? So I changed it. ...this is a really good opportunity to change that story, to change this image of the woman and the associations that get made about her. ...I deliberately sought out the pictures that are softer.

This quote demonstrates not only an understanding of the messages implicit within online information but also a developing sense of agency in participant nine of her ability and obligation to rewrite history.

The editathon provided an opportunity for participants not only to develop their understanding of the historical narratives surrounding women but also equipped them with the skills and evolving agency to actively challenge and rewrite history. While only three interviewees felt comfortable editing Wikipedia entries prior to the editathon event, all nine felt confident to contribute content after the event. Justine reflected on her shift from passive consumer to active contributor:

You know I am much more likely now to go into Wikipedia and think "oh I'll just add a sentence in there about this because it's relevant and appropriate to do so," whereas before I'd just say "oh there's nothing on Wikipedia about this."

This new sense of agency and responsibility was similarly reflected in Anna's comment:

Once I got into the thing on the day I continued to edit pages that I started on that day ...there was one woman who didn't have a page at all and I put her page in there and so now I feel quite motivated to keep going and feel I do have strong ownership.

This sense of agency appeared to develop over the editathon. While at first it was connected to developing confidence and knowledge of how to edit entries, over the course of the editathon, participants' agency was also connected to their understanding of the responsibilities and opportunities being a Wikipedia editor provides for constructing history, or at least accounts of historical events. 
By moving participants from passive consumers of online content to active contributors who have a commitment to, and feel ownership over, the substance and presentation of content on Wikipedia, the editathon developed participants' understanding of the role that the Internet can play in advancing new histories and providing a voice to women and events that otherwise go unrecognised. As Anna explained: "I think we uncovered things which, well while it wasn't new information, it's always been sitting there waiting to be discovered. We uncovered information and brought it out into the light I would say." This quote demonstrates an emerging recognition that history and its presentation on and through digital media are not static. Rather, they are able to be redeveloped, re-mixed, and reoriented in ways that can challenge traditional narratives and perspectives and promote new discourses.

\section{Power of Wikipedia}

Participating in the editathon not only provided participants with a more critical lens for viewing and interpreting information online, but also gave rise to new understandings of how Wikipedia shapes access to information and influences prevailing discourses. Exploring the intersection between Wikipedia and the scholarly and academic conventions and traditions of the university was one of the objectives of the the editathon, as one of the organizers, Marie, explained:

Instead of avoiding Wikipedia and seeing it as a problem and we shouldn't go anywhere near it if we're real academics, it's actually a fascinating and wonderful tool to engage with to develop academic competencies. So that's why I wanted to organise such an event and convert other people to seeing Wikipedia in potentially a different light to how they had been trained to see it.

The editathon provided in participants with insight into the tensions between openness and authority, enabling them to reflect on the role that Wikipedia plays both within their own lives and more broadly in society. Carolyn described Wikipedia as an "extension of [her] memory." Participating in the editathon, however, also caused her to (re)think her positioning towards Wikipedia and how she engaged with it in her role as an academic:

We often refer to Wikipedia, but actually thinking about how it's created, how it's put together is part of the whole digital education change, it's part of how everybody is coming into a more open forms of learning and engagement, more democratic perhaps, although, immediately when I went to my first meeting I learned a lot about how it's not as democratic as it looks.

Participants further developed their understanding of how digital media and the Internet changes the ways in which information is presented, interpreted, and used, and how this in turn alters or influences the construction of history and historical narratives. While information and history has never been static, the Internet enables the adaptation and modification of information, as well as the juxtaposition of multiple narratives at a rate that previously was not achievable. Justine described her new understanding of the fluidity of information in the digital age:

I mean the story is fluid on Wikipedia that's the danger of it, I guess that's the difference between writing a peer reviewed paper isn't it. But yeah it's made me realise the importance of how you do tell that story and how you make it a living part of our history. 
Participating in the editathon also raised participants understanding of the powerful role Wikipedia plays in shaping our engagement with, and access to, information. A Wikipedia entry has become a symbol of legitimacy and value. There exists a tension between the fluidity and transience of digital information and the visible presence this digital information enables. The power of Wikipedia to elevate information is exemplified by Sarah's experience:

I did a quite Google search and within I'd say less than 2 hours of me putting her page in place it was the top hit that came back in Google when I Googled it and I just thought that's it, that's impact right there and the British Medical Journal obituary started dropping down. So that was a moment as well, less connected with the subject matter and more with the power of engaging with that kind of resource.

\section{The Intersection of Traditional Media and Digital Media}

Participating in the editathon prompted participants to reflect on the tensions that exist between traditional media and digital media. These tensions are shaping presentations of history. For many participants, (re)engaging with traditional media, including books and archival material, was one of the highlights of their editathon experience. It provided renewed understanding of what these traditional media offer. As Melanie explained, "there's information in these books that needs to get online and [we] need to put it there." This is further elaborated in the account of participant two who discussed the role of traditional media in a digital age:

I'm very interested in how we take old forms of print and even pre-print and even things from the oral tradition as well. How we take them forward into new media and incorporate them and change them in the process. It's really interesting.

Embedded within participants' accounts is an awareness of the materiality of Wikipedia as a constructed artefact, and the relationship between the physical objects from the archives, the printed history contained within physical books, and the digital representation that ultimately is developed on Wikipedia.

Underpinning the discussions of media and materiality are changing constructions of information and history in a digital age and, more specifically, increasing awareness that if material or information is not in digital form, it does not readily form part of the ongoing historical narrative. As Justine explained in relation to her engagement with 19th century newspapers from the archives during the editathon: "the more that I read in the newspapers about this, the more I just felt like we need to bring this all to life, you know it's all forgotten, it's not there anymore, and it's really important.”

Wikipedia represents a powerful modality and mechanism for bringing to life forgotten information and lost histories. The editathon motivated participants to open up knowledge and make it more accessible. However, the relationships between the physical and the digital are troubling in several ways. It raises issues around copyright, what was referred to by Elizabeth as "kind of locking away your content so no one can see or use them," and also the use of primary secondary data. These issues raise tensions between the new role of the Wikipedia editor and the conventional role of the archivists and librarians involved in the event. 
Participating in the editathon further prompted a growing recognition of the position and role of institutions within these tensions between traditional, non-digital, and digital media. Seven of the nine participants discussed how their increased awareness of digital media, and issues of access and openness of information and resources, was prompting changes in how they approached their work and professional roles at the University. For two participants (Grant and Melanie), these ideas emerged as their most significant learning from the editathon. Grant, a librarian at the University, described the current University copyright policy around its images as "kind of locking away your content so no one can see or use them." His new appreciation for the importance of how media is licensed, and the impact this has on their ability to be accessed online, has prompted him to push for changes to the University's policies.

Marie similarly has used her new understanding of the interplay between primary and secondary evidence to influence her work. She described this learning journey:

one of the real restrictions that we've got is that with Wikipedia you're not allowed to draw on primary data you have to draw on secondary data and so we weren't allowed to use any of the primary data that we had access to. That was a real learning point for me. Actually thinking back on it I didn't expect to learn about it because I didn't know about it ... it's helped me to understand how we need to change or develop as an institution in order to function better in an open educational resource world.

Underpinning the accounts of participants three and five is the appreciation of the potential Wikipedia, and the Internet more generally, has for democratising access to information and challenging the roles of traditional gatekeepers of this information. This notion of possibilities and potentialities of openness, however, is counterbalanced in both participants' narratives by their reflection of how digital media can and does perpetuate traditional inequalities.

\section{Conclusions}

Analysing the narratives of editathon participants' learning journeys, through the lens of the critical approach, provides new insights into the potential of editathons to enable individuals to develop new understandings of the role and power that comes with moving from being a consumer to a producer of knowledge online, and how this impacts on the form, focus, and truth of the information that is disseminated.

Growing into the editor role, the participants recognise their personal responsibility for representing historical people and events that traditionally have been under-represented. As participants' knowledge of the editor role grows, their understanding of the power of social media and the troubled relationship between physical and virtual spaces and histories, as well as, past and present interpretations and representations of people and events, compels them (or at least some of them) to become active in ways they had not foreseen. Here Melluci's (1996) concept of cognitive liberation becomes particularly relevant. The participants' experiences enabled them to connect the abstract ideas about the under and misrepresentation of women and minorities both in contemporary media and in historical discourses, to more tangible examples with which they were actively involved. Participants recognised how new media forms are continuing to perpetrate existing cultural norms 
and inequities, and that by becoming knowledge producers they were in a position to challenge and redress these inequities.

Participants' advocacy for making openly available and accessible forgotten or under-represented histories aligns with the term information activism, which refers to the role, typically of librarians and archivists, of promoting access to, and the removal of, barriers to information. Inherent in this term is the need to expose the structural and systematic biases that exist in the selection, presentation, and dissemination of information. The topic of the editathon provided an opportunity for participants to gain an understanding of the gender disparities that exist in the treatment and presentation of women on Wikipedia. Participation further enabled participants to develop their technical, practical knowledge, and skills in how to edit Wikipedia pages and their induction into the norms and culture of the Wikipedia community, provided the opportunity and the development of agency among participants to actively challenge these biases.

It is important to note that the themes and ideas explored in this paper were not the focus of the interviews. Similarly, as only just over $20 \%$ of editathon participants were interviewed, they represent only a sub-set of those involved. . The analysis presented here, however, does suggest the potential power of editathons as learning events and provides several directions for future research. Designing a study that seeks to capture the narratives of editathon participants as they undertake the journey to becoming editors could provide greater insight into the observations identified in this study.

The design and structure of the editathon as a learning event that combines online activity with offline, in-person collaboration and interaction, and participants' engagement with a range of artefacts and types of media developed in participants' new understandings of issues of materiality, and more particularly, issues of materiality in a digital world. Similarly to the understandings emerging from participants' narratives of the role of the Internet in shaping their access to, and engagement with, information in their everyday lives, participants' accounts also contained reference to notions of sociomateriality. Participants moved from seeing artefacts as discrete objects that convey information to the objects as entangled in complex and dynamic processes that are embedded within their everyday practices and lives (Sorensen, 2009) with each material pattern producing different forms of knowledge.

The learning journeys emerging from the interviews moves beyond traditional conceptions of knowledge as acquisition or transfer, to learning and knowledge as participation within and through interactions with different content, processes, tools, technologies, social relations, and contexts (Fenwick, 2015). Fenwick (2015) suggests that material things are performative; that they act, with other things and forces, to regulate particular forms of participation and to promote particular relations. The editathon developed participants' understanding of how this complex interplay of materials, technology, and social relations is played on Wikipedia and how this in turn influences how consumers engage with information. By becoming contributors rather than just consumers of information on Wikipedia during the editathon event, participants developed new awareness and understanding of Wikipedia as heterogeneous assemblages (Barad, 2007). That is nature, technologies, humanity, and materials act together on Wikipedia to bring forth particular messages and information in our everyday life. 
The adoption of the critical approach in the analysis of the personal narratives of participants' experiences of an editathon event provides a new lens on the range of editathon learning opportunities. By moving beyond the original approach of this study, which was concerned with an understanding of how the construction of social ties between participants facilitated new knowledge construction and learning, this paper has explored the depth of learning and new understandings that an informal learning event can promote. It further suggests the potential and power of an editathon to support new critical understandings and responses, and an evolving sense of agency among participants.

These findings identify valuable learning for improving the study were it to be run with another group. In particular, the range of learning and new knowledge that participants acquired during the editathon suggests that there is potential to further expand the interview schedule in order to more fully capture the development of new understandings that emerged. Future studies would benefit from greater consideration of the qualitative data collection techniques that could be utilised to capture the rich narrative learning emerging from the participants. Conducting interviews prior to the editathon, as well as at its conclusion, would further support the development of rich datasets that more fully captured the journey participants underwent when transitioning from primarily consumers or users of online information to contributors of knowledge, and the factors that supported and impeded this journey.

By focusing on the editathon as an informal, active, experiential learning activity, offers several implications for those involved in the research and delivery of online and distance learning. It reinforces the importance of experiential learning events where participants have the opportunity to shape their own engagement and are not bound by predefined learning intentions or outcomes. Not only did participants develop a greater knowledge of Wikipedia and editing conventions, as the researchers imagined at the beginning of the study, but they also experienced a much deeper learning. It was through the act of moving from consumer to contributor and becoming part of the community of editors, that participants could not only more fully understand issues of bias and structural inequities on Wikipedia, but also actively challenge and address these issues. Furthermore, by negotiating the intersection of traditional, non-digital media with digital media and open source, participants developed new understandings of materiality in a digital age. This suggests that there is substantial learning opportunities if instructional designers consider how they can support learners to take more active roles contributing to online environments. 


\section{References}

Antin, J., Yee, R., Cheshire, C., \& Nov, O. (2011). Gender differences in Wikipedia editing. Paper presented at the ACM WikiSym, Mountain View, California.

Bakardijieva, M., \& Smith, R. (2001). The internet in everyday life: Computer networking from the standpoint of the domestic user. New Media \& Society, 3(1).

doi/10.1177/1461444801003001005

Barad K. (2007). Meeting the universe half-way. Durham, NC: Duke University Press.

Cohen, N. (2011, January 30). Define gender gap? Look up Wikipedia's contributor list. The New York Times Retrieved from https://www.nytimes.com/2011/o1/31/business/media/31link.html

Collier, B., \& Bear, J. (2012). Conflict, criticism, or confidence: An empirical examination of the gender gap in Wikipedia contributions. Proceedings of the ACM 2012 conference on Computer Supported Cooperative Work (pp. 383-392). New York: ACM.

Couldry, N. (2012). Media, society, world: Social theory and digital media practice. London: Polity.

Ebersbach, A. \& Glaser, M. (2004). Towards emancipatory use of a medium: The Wiki. International Journal of Information Ethics, 2(11), 1-9.

Eraut, M. (2000). Non-formal learning and tacit knowledge in professional work. British Journal of Educational Psychology, 70, 113-136.

Fenwick, R. (2015). Sociomateriality and Learning: a critical approach. In D. Scott \& E. Hargreaves (Eds.), The Sage Handbook of Learning. [E-reader Version]. doi: 10.4135/9781473915213.n8

Fenwick, T., Nerland, M., \& Jensen, K. (2012). Sociomaterial approaches to conceptualising professional learning, knowledge and practice (Introduction). Journal of Education and Work, 25(1), 1-13.

Filipacchi, A. (2013, April 27). Wikipedia's sexism. The New York Times. Retrieved from https://www.nytimes.com/2013/04/28/opinion/sunday/wikipedias-sexism.html

Glott, R., Ghosh, R., \& Schmidt, P. (2010). Wikipedia survey: Overview of results. Maastricht: UNUMERIT. Retrieved from http://www.ris.org/uploadi/editor/1305050082Wikipedia_Overview_15March2010FINAL.pdf

Graells-Garrido, E., Lalmas, M., \& Menczer, F. (2015). First women, second sex: Gender bias in Wikipedia. In Proceedings of the 26th ACM conference on hypertext (pp. 165-174). New York: ACM. doi:10.1145/2700171.2791036.

Gunter, H. (2009). The 'C' word in educational research. Critical Studies in Education, 50, 93-102. 
Hargittai, E. \& Shaw, A. (2015). Mind the skills gap: The role of Internet know-how and gender in differentiated contributions to Wikipedia. Information, Communication \& Society, 18(4), 424-442. doi: 10.1080/1369118X.2014.957711

Harp, D., \& Tremayne, M. (2006). The gendered Blogosphere: Examining inequality using network and feminist theory. J\&MC Quarterly, 83(2), 247-264.

Hill, B. M., \& Shaw, A. (2013). The Wikipedia gender gap revisited: Characterizing survey response bias in peer production communities. PLOS ONE, 8(6), e65782. doi:10.1371/journal.pone.0065782

Knorr-Cetina, K. (2007). Culture in global knowledge societies: knowledge cultures and epistemic cultures. Interdisciplinary Science Reviews, 32(4), 361-375.

Knorr-Cetina, K. (2008). Objectual practice. In M. Mazzotti (Ed.), Knowledge as social order: Rethinking the sociology of barry barnes (p.83-98). Hampshire: Ashgate Publishing Limited.

Lam, S. K., Uduwage, A., Dong, Z., Sen, S., Musicant, D. R., Terveen, L., \& Riedl, J. (2011). WP: clubhouse? An exploration of Wikipedia's gender imbalance. In WikiSym 2011 Conference Proceedings - 7th Annual International Symposium on Wikis and Open Collaboration (pp. 110). DOI: $10.1145 / 2038558.2038560$

Littlejohn, A., \& Hood, N. (2018). Becoming an online editor: Perceived roles and responsibilities of Wikipedia editors. Information Research, 23(1).

Manovich, L. (2009). The practice of everyday (Media) life: From mass consumption to mass cultural production? Critical Inquiry, 35(2), 319-331.

Melluci, A. (1996). Challenging codes. Cambridge, UK: Press Syndicate of the University of Cambridge.

Miles, M., \& Huberman, A. (1994). An expanded sourcebook: Qualitative data analysis ( $2^{\text {nd }}$ ed.). Thousand Oaks: Sage.

Oliver, M. (2011). Technological determinism in educational technology research: Some alternative ways of thinking about the relationship between learning and technology. Journal of Computer Assisted Learning, 27, 373-384.

Reagle J., \& Rhue, L. (2011). Gender bias in Wikipedia and Britannica. International Journal of Communication, 5, 1138-1158.

Selwyn, N. (2008). Developing the technological imagination: Theorising the social shaping and consequences of new technologies. In S. Livingstone (Ed.), Theorising the benefits of new technology for youth (pp. 18-28). London: London School of Economics.

Selwyn, N. (2010). Looking beyond learning: Notes towards the critical study of educational technology. Journal of Computer Assisted Learning, 26, 65-73. 
Shaw, A. (2014). The internet is full of jerks, because the world is full of jerks: What feminist theory teaches us about the internet. Communication and Critical/Cultural Studies, 11(3), 273-277.

Sorensen, E. (2009). The materiality of learning: Technology and knowledge in educational practice. Cambridge: Cambridge University Press.

Wagner, C., Graells-Garrido, E., Garcia, D., \& Menczer, F. (2016). Women through the glass ceiling: gender asymmetries in Wikipedia. EPJ Data Science, 5(5), 1-24.

Wikipedia (2016). Wikipedia: Neutral point of view. Retrieved from

https://en.wikipedia.org/wiki/Wikipedia:Neutral point of view

Wikimedia Foundation (2011). Wikipedia Editors Study; Results from the editor survey. Retrieved from https://upload.wikimedia.org/wikipedia/commons/7/76/Editor Survey Report April 2011.pdf 